\title{
ERRATUM
}

\section{Erratum to : The first African record of Artolenzites acuta comb. nov. (Basidiomycota, Polyporaceae)}

\section{Ambit RT ${ }^{1}$ and Mossebo DC ${ }^{1^{*}}$}

${ }^{1}$ University of Yaoundé 1, Mycological Laboratory, B.P. 1456 Yaoundé, Cameroon

Ambit RT, Mossebo DC 2015 - The first African record of Artolenzites acuta comb. nov. (Basidiomycota, Polyporaceae). Mycosphere 6(4), 401, Doi 10.5943/mycosphere/6/4/1

Erratum to : Mycosphere 6(3), 280-289, Doi 10.5943/mycosphere/6/3/6

The original publication contains the following errors:

Page 282, line 34: Delete the words

Lenzites acuta Berk (1842)

Dadalea inaequabilis Berk (1843)

Daedalea flavida Lev. L.C. (1844)

Trametes lobata Berk (1851)

Lenzites becklerii Berk (1872)

Daedalea isabellina Murr. (1908)

Lenzites adusta Mass (1910)

Hexagonia flavofusca Lloyd (1922)

It should be read as

- $\quad$ Basionym

Lenzites acuta Berk. London J. Bot. 1(3): 146 (1842) ( MBキ200258)

- $\underline{\text { Synonyms }}$

Cellulariella acuta (Berk.) Zmitr. Et Malysheva, IF 180 : 1 (2014) (MB $\neq 804637)$

Cellularia acuta (Berk.) Kuntze, Revisio generum plantarum 3 : 451 (1898) (MB $\neq 468434)$

Trametes acuta (Berk.) Imazeki, Bulletin of the Tokyo Science Museum 6: 73 (1943) $(\mathrm{MB} \neq 282909)$

The online version of the original article can be found at http://mycosphere.org/pdfs/Mycosphere_6_3_6.pdf 